\title{
Description of the First Strain of 2019-nCoV, C-Tan-nCoV Wuhan Strain - National Pathogen Resource Center, China, 2020
}

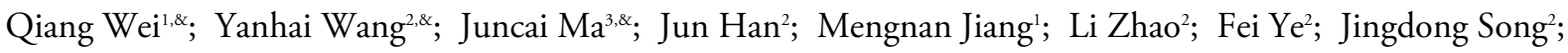 \\ Bo Liu'; Linhuan Wu ${ }^{3}$; Wenjie Tan ${ }^{2, *}$; Guizhen Wur, ; George F. Gao'; Jianjun Liu ${ }^{1, *}$
}

The recent outbreak of novel coronavirus-infected pneumonia (NCIP) in China (1) has attracted much attention domestically and internationally. As the outbreak occurred, China carried out emergency responses quickly, notified the World Health Organization (WHO) in a timely manner, and shared the viral gene sequence with international communities immediately after pathogenic identification.

The timely release of information on the novel coronavirus (2019-nCoV), joint research on relevant viral genome mutations, pathogenicity, and transmission mechanisms, vaccine development, and drug screening and evaluation are of great significance for epidemic prevention and control. Therefore, for prevention and control of the NCIP epidemic and for future scientific research on 2019-nCoV, the National Pathogen Resource Center (NPRC) published information of the first strain of $2019-\mathrm{nCoV}$ on the National Science and Technology Resource System for Novel Coronavirus (http://nmdc.cn/\#/nCoV) on January 24, 2020.

\section{Virus Isolation}

Researchers from the National Institute for Viral Disease Control and Prevention (IVDC) of China CDC successfully isolated the virus out of lower respiratory alveolar lavage fluid from patients with unexplained viral pneumonia in Wuhan and detected typical electron microscopic coronavirus morphology on January 6, 2020, which substantiated the successful isolation and culture of the first $2019-\mathrm{nCoV}$ strain in China (2).

\section{Strain Basic Information}

Basic strain information of 2019-nCoV is important basis for downstream sharing, use, research, and epidemic prevention and control. With in-depth study of its biological traits and pathogenicity, further information will be supplemented gradually. (Table 1)
TABLE 1. Basic information for the first strain of 2019nCoV.

\begin{tabular}{ll}
\hline \multicolumn{1}{c}{ Descriptors } & \multicolumn{1}{c}{ Description } \\
\hline Code & CHPC 2020.00001, NPRC 2020.00001 \\
Name in Chinese & 新型冠状病毒武汉株01 \\
Name in English & C-Tan-nCoV Wuhan Strain \\
Taxonomy & Novel $\beta$ genus coronavirus \\
Source of the Specimen & Clinical Patients \\
Source of Collection & Wuhan, Hubei Province, China \\
Isolation Date & Jan 6, 2020 \\
Risk Level & BSL-3 \\
Contact Info & ivdcolm@ivdc.chinacdc.cn, \\
\end{tabular}

Note: CHPC refers to the Center for Human Pathogen Collection of China CDC, and NPRC refers to the National Pathogen Resource Center of China.

\section{Specific Primers and Probes for Detecting 2019-nCoV (3)(Table 2)}

TABLE 2. Real-time RT-PCR. The open reading frame $1 \mathrm{ab}$ (ORF1ab) and the nucleoprotein $\mathrm{N}$ gene region of the virus were recommended for primers and probes.

\begin{tabular}{ll}
\hline \multicolumn{1}{c}{ Item } & \multicolumn{1}{c}{ Sequence } \\
\hline $\begin{array}{l}\text { Target 1 (ORF1ab) } \\
\text { Forward primer (F) }\end{array}$ & CCCTGTGGGTTTTACACTTAA \\
Reverse primer (R) & $\begin{array}{l}\text { ACGATTGTGCATCAGCTGA } \\
\text { 5'-FAM-CCGTCTGCGGTATGTGGAA } \\
\text { AGGTTATGG-BHQ1-3' }\end{array}$ \\
Target 2 (N) & $\begin{array}{l}\text { GGGGAACTTCTCCTGCTAGAAT } \\
\text { Forward primer (F) }\end{array}$ \\
Reverse primer (R) & $\begin{array}{l}\text { CAGACATTTTGCTCTCAAGCTG } \\
\text { 5'-FAM-TTGCTGCTGCTTGACAGATT- } \\
\text { TAMRA-3' }\end{array}$ \\
\hline
\end{tabular}

Note: Results reading: negative (no $\mathrm{Ct}$ value or $\mathrm{Ct}=40$ ); positive ( $\mathrm{Ct}$ value $<37$, considered positive); and suspicious (if $\mathrm{Ct}$ value is between 37 and 40 , repeat testing is recommended). If $\mathrm{Ct}$ value $<40$ in repeat testing and distinctive peaks are observed in the amplification curve, the sample is considered positive; otherwise the sample is considered negative. 


\section{Strain Imaging}

Negatively stained 2019-nCoV particles generally present a spherical shape with some pleomorphism. The viral particles range from $60-140 \mathrm{~nm}$ in diameter. Discernable spikes of $9-12 \mathrm{~nm}$ on the envelope render the virions appearance of a solar corona (hence named coronavirus) (Figure 1).

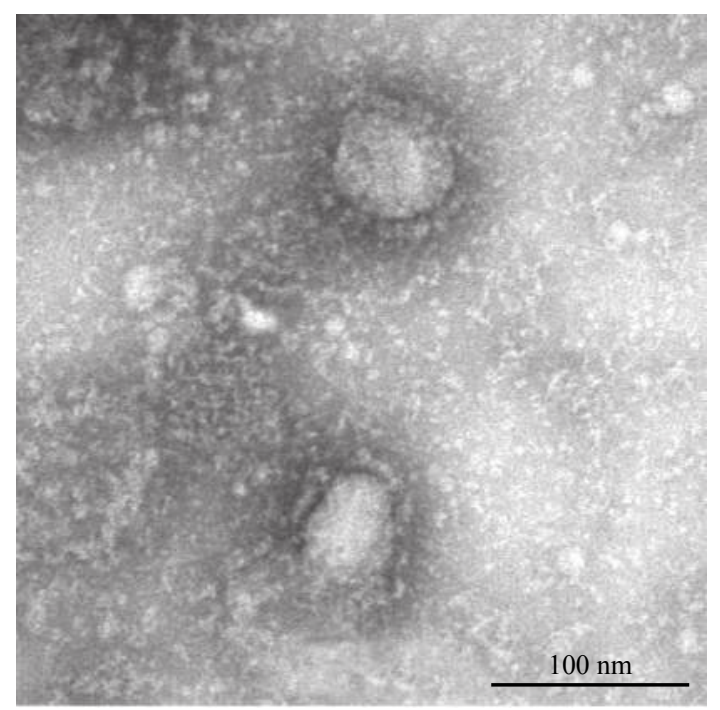

FIGURE 1. Negatively stained 2019-nCoV particles in the supernatant of infected cells culture.

\section{Strain Sharing}

The 2019-nCoV strains can be shared for external use under proper administrative approvals and specified conditions. Users must indicate the source of the strains on relevant materials, including but not limited to publications and awards, as the following: National Pathogen Resource Center (National Institute for Viral Disease Control and Prevention, China CDC) with the NPRC number. Upon completion of the specified activities, users should return the strains to NPRC (IVDC) or perform proper destruction and disposal and issue a description of use to NPRC.

NPRC is a national science and technology resource sharing service platform established by China CDC. As a National Scientific and Technological Innovation Center, it is mainly responsible for the collection, identification, labeling, preservation, and provision of national pathogenic microbial resources. Through systemic integration of pathogenic microbial resources and enhancement of utility efficiency, NPRC is contributing to scientific and technological development as well as social welfare with its lasting conviction for a sharing and open society.

\section{Acknowledgements}

This work was supported by National Science and Technology Major Project on Important Infectious Diseases Prevention and Control (2018ZX10734404) and National Key Research and Development Project (2020YFC0840800, 2020YFC0840900).

\# Corresponding authors: Guizhen Wu, wugz@ivdc.chinacdc.cn; WenjieTan, tanwj@ivdc.chinacdc.cn; Jianjun Liu, Liujj@chinacdc.cn.

\footnotetext{
${ }^{1}$ National Pathogen Resource Center, Chinese Center for Disease Control and Prevention, Beijing, China; ${ }^{2}$ NHC Key Laboratory of Biosafety, National Institute for Viral Disease Control and Prevention, China CDC, Beijing, China; ${ }^{3}$ National Microbiology Data Center, Institute of Microbiology, Chinese Academy of Science, Beijing, China.

\& Joint first authors.
}

Submitted: January 24, 2020; Accepted: January 29, 2020

\section{References}

1. Tan WJ, Zhao X, Ma XJ, Wang WL, Niu PH, Xu WB, et al. A novel coronavirus genome identified in a cluster of pneumonia cases Wuhan, China 2019-2020. China CDC Weekly 2020;2(4):61-2. http://weekly.chinacdc.cn/en/article/ccdcw/2020/4/61.

2. Zhu N, Zhang DY, Wang WL, Li LW, Yang B, Song JD, et al. A novel coronavirus from patients with pneumonia in China, 2019. N Engl J Med. [2020-1-24]. http://dx.doi.org/10.1056/NEJMoa2001017.

3. Institute of Virus Disease Control and Prevention, China CDC. Primers and probes for detection of the novel coronavirus. [2020-1-21]. http:// ivdc.chinacdc.cn/kyjz/202001/t20200121_211337.html. (In Chinese). 